\title{
因HAD
}

ISSN-L: 2530-5115

DOI: http://doi.org/10.22585/hospdomic.v2i1.33

\section{Adherencia a la Nutrición Enteral Domiciliaria}

\section{Adherence to Home Enteral Nutrition}

Carmina Wanden-Berghe', Kamila Cheikh Moussa², Javier Sanz-Valero ${ }^{3}$

1 Hospital General Universitario de Alicante; Instituto de Investigación Sanitaria y Biomédica de Alicante (ISABIAL), Alicante, España.

2 Instituto de Investigación Sanitaria y Biomédica de Alicante ISABIAL - FISABIO, Alicante, España.

3 Universidad Miguel Hernández, Sant Joant d’Alacant, Alicante, España.

Correspondencia/Correspondence

Carmina Wanden-Berghe

carminaw@telefonica.net

Recibido/Received

15.11 .2017

Aceptado/Accepted

12.01 .2018
Conflicto de Intereses/Competing interest No existe ningún conflicto de interés en el presente estudio.

\section{Agradecimientos/Acknowledgments}

Este trabajo es parte de la tesis doctoral de Al Cheikha Kamela Cheikh Moussa y ha contado para su desarrollo con una beca concedida por VEGENAT HEALTHCARE. 


\section{RESUMEN}

Objetivo: Elaborar un cuestionario que permita evaluar la adherencia terapéutica de los pacientes con nutrición enteral domiciliaria (NED).

Método: Generación y validación de un cuestionario para evaluar la adherencia a la NED. Los ítems se determinaron mediante consenso se expertos. La consistencia interna se comprobó mediante el estadístico de Kuder-Richardson Formula 20. La validez de constructo fue analizada con el método de Rotación Varimax con normalización de Kaiser. La concordancia intra-observador e inter-observador se calculó mediante el índice de Kappa.

Resultados: Se determinaron 6 ítems de respuesta dicotómica (si.no) mediante el consenso agrupados en dos categorías: conocimientos y dificultades. Estos ítems ofrecieron una puntuación final relacionada con la adherencia de 0 a 2 = baja; 3 a 4 = moderada; 5 a 6 = alta. La fiabilidad intra-observador obtuvo una concordancia igual a 1 e inter-observadores de 0,93. La consistencia interna fue de 0,721. La validez de constructo analizada mediante un análisis factorial por análisis de componentes principales con método de Rotación Varimax con normalización de Kaiser, identificó 2 componentes principales que agruparon a los ítems en las dos dimensiones del cuestionario.

Conclusión: El cuestionario de adherencia nutricional desarrollado se ha mostrado fiable y útil para obtener la información sobre adherencia al tratamiento nutricional, observándose alta validez de contenido, consistencia interna, validez de constructo y estabilidad.

Palabras clave: Encuestas y Cuestionarios; Cumplimiento de la Medicación; Estudios de Validación; Nutrición Enteral.

\section{ABSTRACT}

Objective: To develop a questionnaire that allows to evaluate the therapeutic adherence of the patients with home enteral nutrition (NED).

Method: Generation and validation of a questionnaire to evaluate medication adherence to NED. The internal consistency was verified by the Kuder-Richardson Formula 20 statistics. The construct validity was analyzed with the Varimax Rotation method with Kaiser normalization. The intra-observer and inter-observer concordance was calculated using the Kappa index.

Results: Six items of dichotomous response were determined (yes.no) by consensus grouped into two categories: knowledge and difficulties. These items offered a final score related to the adherence from 0 a 2 = low; 3 a $4=$ moderate; 5 a 6 = high. The intra-observer reliability obtained a concordance equal to 1 and inter-observers 0.93. The internal consistency was 0.721 . Construct validity analyzed by factor analysis by principal component with Varimax Rotation method with Kaiser normalization identified 2 main components that grouped the items in the two dimensions of the questionnaire.

Conclusion: The nutritional adherence questionnaire developed has been shown to be reliable and useful in obtaining information on adherence to nutritional treatment, with high content validity, internal consistency, construct validity and stability.

Keywords: Surveys and Questionnaires; Medication Adherence; Validation Studies; Enteral Nutrition. 


\section{INTRODUCCIÓN}

La eficacia de un tratamiento va a venir determinada por varios factores; unos son intrínsecos a la propia eficacia del tratamiento, otros derivados de la enfermedad y otros que vienen determinados por la adherencia al tratamiento (1-2).

La preocupación por la adherencia al tratamiento cobró importancia en la segunda mitad del siglo XX, cuando los avances de las ciencias médicas hicieron que los tratamientos fueran más seguros y eficaces, pero donde la falta de cumplimiento seguía generando la no resolución del motivo de indicación y la aparición de problemas concomitantes. Esta compleja situación continúa siendo un problema vigente, probablemente porque en los últimos años se ha evidenciado un aumento progresivo de la incidencia de enfermedades crónicas (3) que conllevan la coexistencia de múltiples y variados tratamientos simultáneos en un mismo paciente por un prolongado periodo (1).

El concepto y la definición de adherencia ha venido siendo discutida y controvertida (4-6) por lo que la Organización Mundial de la Salud (OMS) emitió un informe en junio del 2003 sobre «Adherencia a terapias a largo plazo» donde pone de manifiesto que la definición considerada hasta entonces (5) se centra estrictamente en la adherencia al tratamiento farmacológico o las indicaciones médicas, y que no contempla ningún otro tipo de intervención o recomendación respecto a cambios sobre estilos de vida (higiene, ejercicio, alimentación, nutrición, etc.) quedando obviados un gran número de comportamientos del paciente que comprometen la evolución de su patología y de su bienestar. Por lo que la OMS propuso en el Adherence Project (7) actualizar el concepto como «El grado en que el comportamiento de una persona al tomar un medicamento, seguir un régimen alimentario y realizar cambios en su estilo de vida se corresponda con las recomendaciones acordadas con su referente sanitario».

Las sociedades europea y americana de nutrición enteral y parenteral pusieron de manifiesto, en sus guías clínicas, la existencia de discrepancias entre las cantidades prescritas y las recibidas por los pacientes a los que se les administró nutrición artificial por sonda (8,9). Estudios en el entorno hospitalario han tratado de evaluar la adherencia durante el periodo de ingreso (10). Aunque, estos resultados puedan considerarse como una aproximación a la valoración de la adherencia no se puede suponer que con ellos se tiene una visión real, ni siquiera aproximada de lo que sucede con los pacientes que tienen prescrita nutrición enteral por tiempo prolongado, sobre todo si se refiere al entorno domiciliario, donde los controles, registros, bombas, dedicación e interés porque se cumpla el régimen terapéutico nutricional establecido es, sin duda, de lo más variable.

No se ha identificado ninguna herramienta dirigida a la valoración de la adherencia terapéutica al tratamiento nutricional enteral, siendo éste uno de los principales tratamientos en más de la mitad de los pacientes crónicos. Parece razonable y necesario disponer de algún instrumento validado que permita tener un control de la adherencia nutricional. En este estudio se propone el desarrollo de un cuestionario para evaluar la adherencia a la nutrición enteral de los pacientes en el entorno domiciliario.

\section{MATERIAL Y MÉTODO}

Para la construcción del cuestionario se procedió a realizar una búsqueda bibliográfica, utilizando los descriptores Medical Subject Headings (MeSH): "Medication Adherence" y "Enteral Nutrition" con los que se construyeron las ecuaciones de búsqueda para su uso en las bases de datos MEDLINE, a través de PubMed, CUIDEN, SCOPUS y la Web of Science, utilizando conectores booleanos. Se empleó el filtro "humans" y la búsqueda se restringió a los últimos siete años, como 
periodo de obsolescencia establecido para las publicaciones en el campo de ciencia de la salud y nutrición (11-13). Partiendo de los artículos identificados se elaboró una propuesta de cuestionario.

Para evaluar la «validez de contenido» se realizó una entrevista con expertos en nutrición e investigación en la que se definieron los conceptos. Se procedió a la verificación de los mismos en una muestra de 30 pacientes. A continuación, se formularon los diferentes ítems para evaluar la adherencia del paciente a su tratamiento nutricional que se agruparon bajo tres dimensiones. Para establecer la validez de constructo se solicitó al grupo de expertos que puntuaran cada uno de los ítems ( 0 = muy poco relevante hasta $10=$ muy relevante) valorando la pertinencia, relevancia, corrección e inteligibilidad, aportando comentarios y sugerencias. El criterio para conservar cada pregunta fue obtener una puntuación media superior a 6 y que presentaran un coeficiente de variación inferior al $25 \%$.

La validez interna se evaluó mediante una prueba piloto con los 30 sujetos para determinar la claridad de las preguntas y se determinó la consistencia interna mediante el estadístico de KuderRichardson Formula 20 (KR 20). La validez de constructo fue analizada mediante un análisis factorial por análisis de componentes principales con método de Rotación Varimax con normalización de Kaiser. La validez de criterio externo no pudo ser evaluada al no disponer de un gold estándar que permitiese el análisis.

La estabilidad del cuestionario se evaluó en el grupo de 30 sujetos realizando el cuestionario en dos ocasiones con un periodo de descanso mínimo de 7 días para evitar el sesgo de memoria. La concordancia intra-observador por el mismo encuestador y la concordancia inter-observador por dos encuestadores. Se calculó el índice de Kappa, considerando > 60\% como buena concordancia (14).

\section{RESULTADOS}

La búsqueda bibliográfica localizó 632 artículos y tras aplicar los criterios de inclusión y exclusión se seleccionaron 6 estudios (figura 1) en los que se identificaron 9 cuestionarios y 5 escalas validadas para el cumplimiento del tratamiento farmacológico. No se encontró ningún cuestionario o método de evaluación de la adherencia al tratamiento nutricional.

Se desarrolló un primer borrador de tres bloques de preguntas que tras la primera evaluación de contenido y constructo, y siguiendo la opinión de los expertos se eliminaron los ítems sobre la conducta del paciente o su cuidador y un cuarto ítem de dificultades, al considerar que dicha información queda recogida en las otras dos dimensiones (conocimientos y dificultades del paciente), esto dio paso a un cuestionario con dos bloques de preguntas y 6 ítems dicotómicos (si.no); ver tabla 1.

En los dos bloques de preguntas, la evaluación de los expertos, sobre la pertinencia de los ítems propuestos, todos obtuvieron una puntuación por encima de los 6 puntos (media 8,72 $\pm 1,32$ ). El coeficiente de variación obtenido osciló entre 11,4\% en el ítem D3 y 21,1\% en el C2. Se estableció una puntuación para cada pregunta, Clasificando al paciente con «baja adherencia al tratamiento» entre 0 o 2 puntos, «adherencia media» de 3 o 4 puntos y "alta adherencia al tratamiento» entre 5 y 6 puntos. Permitiendo identificar el motivo de la adherencia en el desconocimiento o en la falta de cumplimiento al evaluarse los dos bloques por separado (ver tabla 1).

La fiabilidad intra-observador obtuvo una concordancia entre las dos observaciones con un índice de Kappa igual a 1 y la fiabilidad inter-observadores Kappa de 0,93. La consistencia interna mediante el estadístico KR (20) fue de 0,721. La validez de constructo analizada mediante un análisis factorial por análisis de componentes principales con método de Rotación Varimax con 
normalización de Kaiser, identificó 2 componentes principales que agrupó a los ítems en las dos dimensiones definidas por el cuestionario.

Figura 1. Diagrama de flujo de la selección de artículos

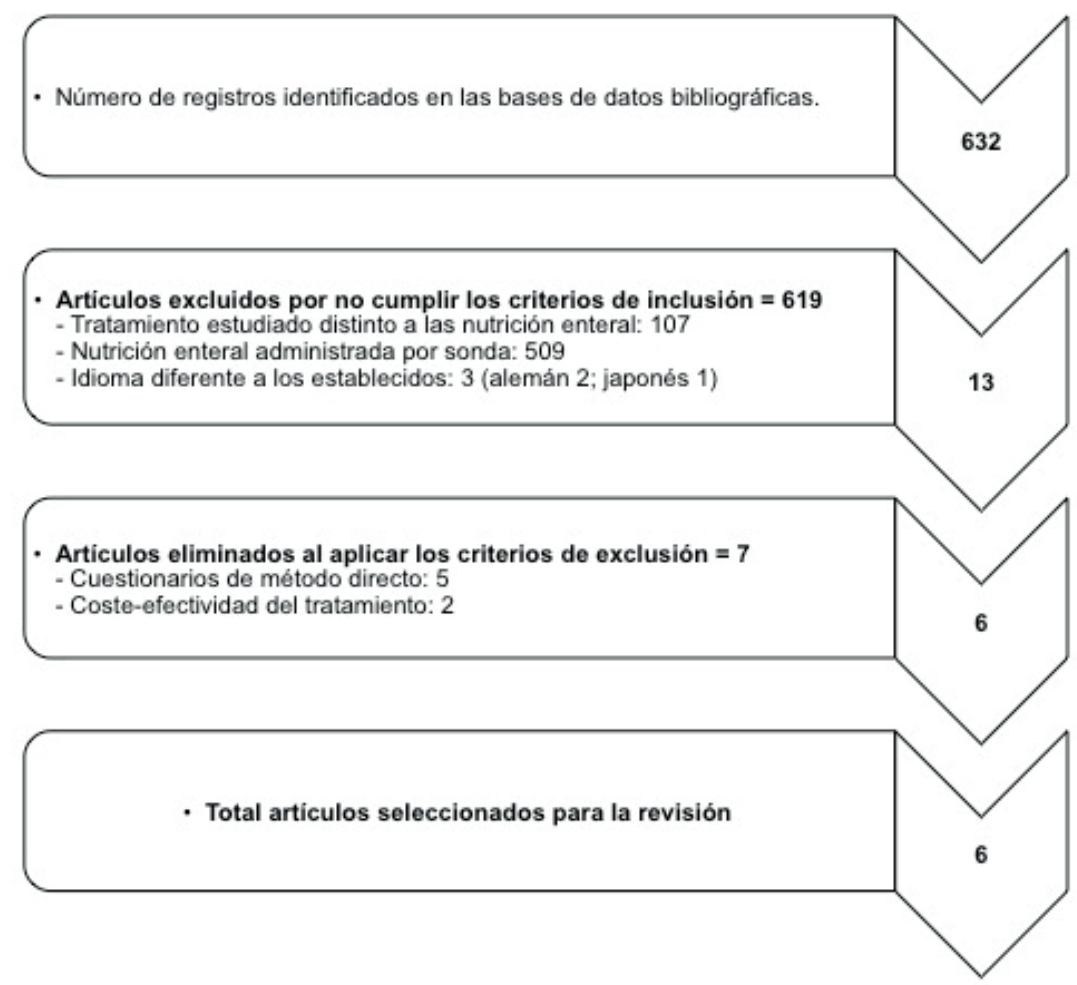


Tabla 1. Cuestionario de Adherencia al Tratamiento Nutricional

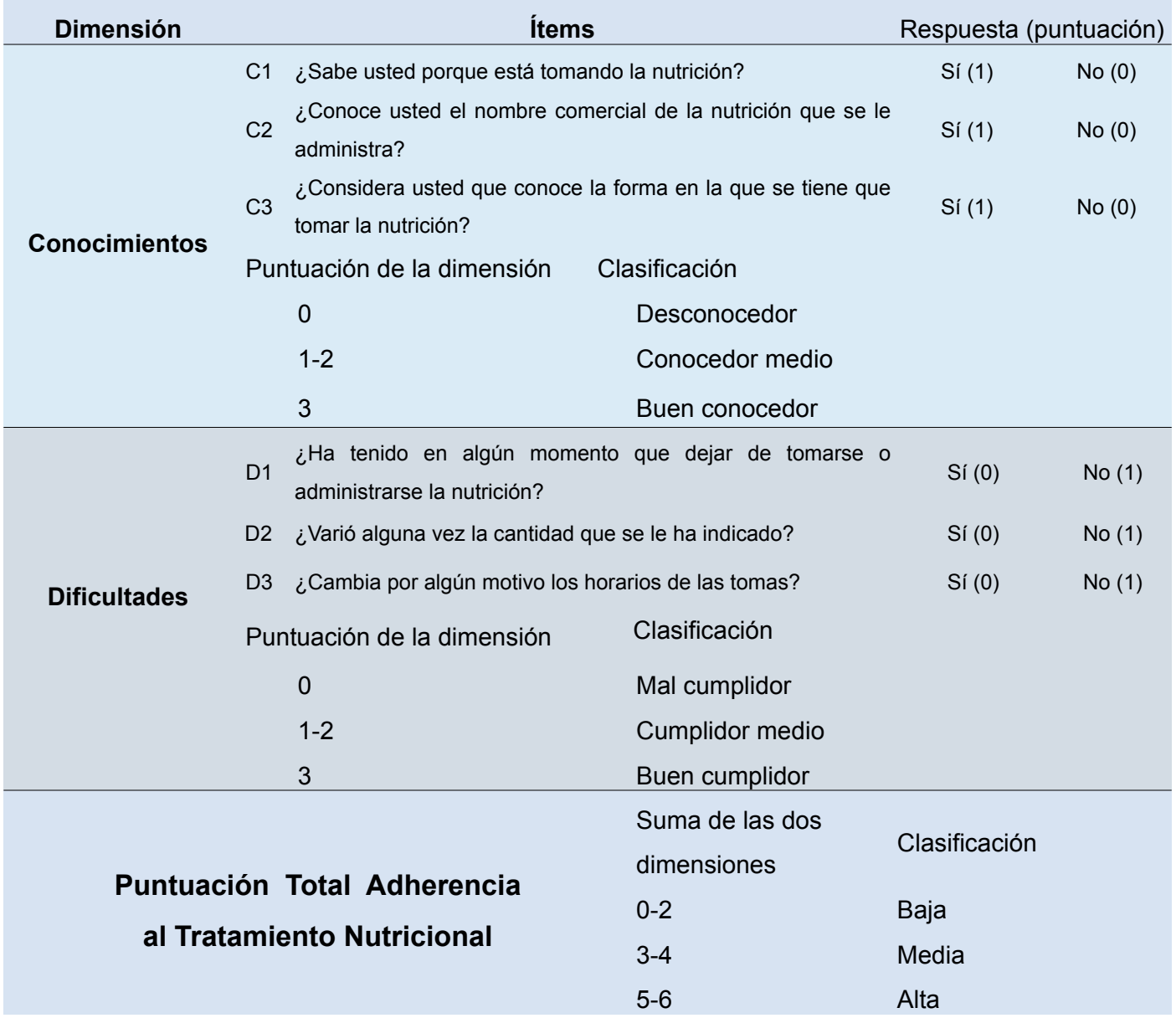




\section{DISCUSIÓN}

Es de resaltar el carácter novedoso del presente trabajo ya que hasta este momento no se había propuesto un cuestionario normalizado para medir la adherencia al tratamiento nutricional, aun siendo una necesidad reivindicada por la Organización Mundial de la Salud a través de sus políticas iniciadas en el año 2001 sobre la adherencia en las terapias a largo plazo $(7,15)$. A esto, se le suma que en los últimos trabajos sobre adherencia terapéutica, únicamente se encontraron herramientas estandarizadas para el tratamiento farmacológico, mientras que para el estudio del cumplimiento en la ingesta de suplementos nutricionales y nutrición enteral se siguen utilizando métodos indirectos no estandarizados (16); encontrando algunos trabajos que utilizan el conteo del número de envases consumidos, medir la cantidad de producto restante para determinar las calorías ingeridas al día y otros como es el uso de registros o diarios dietéticos de cinco días, métodos útiles pero no libres de sesgos importantes como es introducir en los resultados la propia sobreestimación del paciente (16-18).

La elaboración de este cuestionario, aunque se trata de un método indirecto de medida de la adherencia, ya que no se dispone de métodos inmediatos de monitorización de la ingesta que sean accesibles, se basó en los factores más influyentes sobre el resultado de la terapia recibida (19); el grado de conocimiento de la enfermedad por el paciente, causa por la que está recibiendo la nutrición y su suficiencia para seguir las indicaciones recibidas.

El instrumento está dotado de validez racional al haber utilizado el conocimiento previo sobre adherencia terapéutica y además se ha conseguido en su creación una buena validez de contenido y validez de constructo al contemplar las dos dimensiones determinantes de la adherencia, los resultados de la consistencia interna, la alta fiabilidad y el coeficiente de correlación en el test-retest indica que en las dos ocasiones los sujetos han quedado ordenados de la misma o parecida manera. Este hecho podemos entenderlo como un indicador de estabilidad o de no ambigüedad del cuestionario. Por todo ello, probablemente constituya un instrumento estandarizado útil para determinar el grado de adherencia al tratamiento nutricional y que permita desarrollar estrategias de seguimiento e intervención para mejorar la adherencia de los pacientes a este tratamiento.

Conocer la adherencia al tratamiento es determinante para el resultado terapéutico. Y, en consecuencia, se puede concluir que el cuestionario de adherencia nutricional se ha mostrado fiable y útil para obtener la información sobre adherencia al tratamiento nutricional, observándose alta validez de contenido, consistencia interna, validez de constructo y estabilidad.

\section{BIBLIOGRAFÍA}

1. Rodríguez-Marín J, Martínez M y Valcárcel MP. Psicología Social y Psicología de la Salud. En: Rodríguez-Marín J, coordinador. Aspectos psicosociales de la salud y la comunidad. Barcelona, España: PPU Editorial; 1990.

2. Bishop GD. Health Psychology: Integrating Mind and Body. Boston, USA: Allyn and Bacon; 1994.

3. World Health Organization. The World Health Report 2002: Reducing Risks, Promoting Healthy Life. Geneva, Switzerland: World Health Organization; 2002.

4. Meichenbaum D, Turk D. Facilitating treatment adherence. Nueva York, USA: Plenum Press; 1987. 
5. Haynes RB, Sackett DL. Compliance in health care. 2nd edition. Baltimore, USA: Johns Hopkins University Press; 1979.

6. Rand CS. Measuring adherence with therapy for chronic diseases: implications for the treatment of heterozygous familial hypercholesterolemia. Am J Cardiol. 1993;72(10):68-74D. DOI: 10.1016.0002-9149(93)90014-4; PMID: 8213501

7. Sabaté E, editor. Adherence to long-term therapies: evidence for action. Geneva, Switzerland: World Health Organization; 2003.

8. ASPEN Board of Directors and the Clinical Guidelines Task Force. Guidelines for the use of parenteral and enteral nutrition in adult and pediatric patients. JPEN J Parenter Enteral Nutr. 2002;26(1):1SA-138SA. PMID: 11841046

9. Howard P, Jonkers-Schuitema C, Furniss L, Kyle U, Muehlebach S, Od-lund-Olin A, et al. Managing the patient journey through enteral nutritional care. Clin Nutr. 2006;25(2):187-95. DOI: 10.1016.j.clnu.2006.01.013; PMID: 16697502

10. van den Broek PWJH, Rasmussen-Conrad EL, Naber AHJ, Wanten GJA. What you think is not what they get: significant discrepancies between prescribed and administered doses of tube feeding. Br J Nutr. 2009;101(1):68-71. DOI: 10.1017.S0007114508986852; PMID: 18768101

11. Villar Alvarez F, Estrada Lorenzo JM, Peréz Andrés C, Rebollo Rodrí-guez MJ. Estudio bibliométrico de los artículos originales de la Revista Española de Salud Pública (1991-2000). Parte tercera: análisis de las referencias bibliográficas. Rev Esp Salud Pública. 2007;81(3):247-59. DOI: 10.1590.S1135-57272007000300002; PMID: 17694632

12. Castera VT, Sanz Valero J, Juan-Quilis V, Wanden-Berghe C, Culebras JM, García de Lorenzo y Mateos A, et al. Estudio bibliométrico de la revista Nutrición Hospitalaria en el periodo 2001 a 2005: parte 2, análisis de consumo; las referencias bibliográficas. Nutr Hosp. 2008;23(6):541-6. PMID: 19132261

13. Sanz-Valero J, Wanden-Berghe C. Análisis bibliométrico de la producción científica, indizada en MEDLINE, sobre los servicios de salud proporcionados por las unidades de hospitalización a domicilio. Hosp Domic. 2017; 1(1):21-34. DOI: 10.22585.hospdomic.v111.3

14. Landis JR, Koch GG. The measurement of observer agreement for categorical data. Biometrics. 1977;33(1):159-74. DOI: 10.2307.2529310; PMID: 843571

15. Sabaté E. WHO Adherence Project: Toward Policies for Action. Geneva, Switzerland: World Health Organization; 2001.

16. Hubbard GP, Buchan B, Sanders K, Brothers S, Stratton RJ. Improved compliance and increased intake of energy and protein with a high energy density, low volume multi-nutrient supplement. Proc Nutr Soc. 2010;69(OCE2):E164. DOI: 10.1017.S0029665109993600

17. Gianotti L, Braga M, Nespoli L, Radaelli G, Beneduce A, Di Carlo V. A randomized controlled trial of preoperative oral supplementation with a specialized diet in patients with gastrointestinal cancer. Gastroenterology. 2002;122(7):1763-70. DOI: 10.1053.gast.2002.33587; PMID: 12055582

18. Fearon KCH, Von Meyenfeldt MF, Moses AGW, Van Geenen R, Roy A, Gouma DJ, et al. Effect of a protein and energy dense $\mathrm{N}-3$ fatty acid enriched oral supplement on loss of weight and lean tissue in cancer cachexia: a randomised double blind trial. Gut. 2003;52(10):1479-86. DOI: 10.1136. gut.52.10.1479; PMID: 12970142

19. Basterra Gabarró M. El cumplimiento terapéutico. Pharm Care Esp. 1999;1:97-106. 